\title{
Estimating the binary fraction of planetary nebulae central stars
}

\author{
D. Douchin ${ }^{1,2,3}$, O. De Marco ${ }^{1,2}$, D. J. Frew ${ }^{1,2}$, G. H. Jacoby ${ }^{4}$, \\ J.-C. Passy ${ }^{5,11}$, T. Hillwig ${ }^{6}$, S. B. Howell ${ }^{7}$, H. Bond ${ }^{8}$, A. Peyaud ${ }^{1}$, \\ A. Zijlstra ${ }^{9}$, R. Napiwotzki ${ }^{10}$, G. Jasniewicz ${ }^{3}$ and Q. Parker ${ }^{1,2}$ \\ ${ }^{1}$ Macquarie University Research Centre in Astronomy, Astrophysics \& Astrophotonics \\ ${ }^{2}$ Department of Physics \& Astronomy, Macquarie University Australia ${ }^{3}$ GRAAL, Université \\ Montpellier 2, France ${ }^{4}$ Giant Magellan Telescope,USA ${ }^{5}$ University of Victoria, Canada \\ ${ }^{6}$ Valparaiso University, USA ${ }^{7}$ NASA Ames Research Center, USA ${ }^{8}$ Space Telescope Science \\ Institute, USA ${ }^{9}$ University of Manchester, UK ${ }^{10}$ University of Hertfordshire, UK ${ }^{11}$ American \\ Museum of Natural History, New York City, USA

\begin{abstract}
During the past 20 years, the idea that non-spherical planetary nebulae might need a binary or planetary interaction to be shaped was discussed by various authors. It is now generally agreed that the varied morphologies of planetary nebulae cannot be fully explained solely by single star evolution. Observationally, more binary central stars of planetary nebulae have been discovered, opening new possibilities to understand the connections between binarity and morphology. So far, $\simeq 45$ binary central stars of planetary nebulae have been detected, most being close systems detected via flux variability. In order to determine the PN binary fraction, one needs a method that can detect wider binaries. We present here recent results concentrating on binary infrared excess observations aimed at detecting binaries of any separation.
\end{abstract}

Keywords. techniques: radial velocities, techniques: photometric, binaries: general, stars: statistics, planetary nebulae: general

\section{Introduction}

There is no general concensus yet about what shapes planetary nebulae (PNe). The idea that the presence of a stellar or substellar companion is needed to break the spherical symmetry and account for the observed geometries, called the Binary Hypothesis (for a review, see De Marco 2009), is still being tested. In this context, a number as high as $80 \%$ (Jacoby et al. 2010) of non-spherical PNe potentially implies that the proportion of binary stars in the center of $\mathrm{PNe}$ is much larger than what is currently thought, therefore indicating that PNe could be preferentially created via a binary channel. If the observed fraction of binary central stars of PNe were higher than expected in the current paradigm $(35 \%$ for binaries with separations $<500 \mathrm{AU})$, this would support the idea that PNe are preferentially a binary phenomenon. To estimate the binary fraction, we need an unbiased sample of PNe. The determination of whether a central star is binary is not trivial and requires a suite of methods. In the following, we describe these methods. We also present new results from recent observations and how this constrains the binary fraction in PNe. We conclude by some perspectives to refine this number.

\section{Methods to investigate binarity}

We here present the main methods used to investigate binarity among our PN sample: flux variability, spectral variability and infrared (IR) excess, we also present some recent results obtained by applying these methods. 
The flux variability method is based on the variation of brightness of the unresolved binary system. The three main causes of flux variability are eclipses, tidal deformations but mainly irradiation effects from the hot companion onto the cold one. This method gives a close binary fraction of about 15-20\% (see Bond 2000, Miszalski et al. 2009). It is biased to short periods as the three causes of flux variation all diminish with increasing separation. It is also biased to intrinsically faint stars to avoid periodic variability caused by stellar winds. New discoveries will be possible thanks to Kepler, whose field includes 6 PNe, including Kronberger 61, newly imaged by us in March 2011 (see http://www.gemini.edu/node/11656). Five of the six objects show flux variability (see Douchin et al. these proceedings)

Spectroscopic variability is used to detect the motion of binary systems around their barycenter. Binaries with periods up to a month could potentially be detected with this method. Intrinsically faint stars, with weak or no wind, are targets of choice. This introduces a bias and imposes serious technical restrictions to obtain a sufficient signalto-noise ratio, ranking this method down for statistical purposes required in our case. Out of 7 central stars targeted by the VLT/UVES, we fond that one, A 14, has a radial velocity (RV) variability confirmed at a $3 \sigma$ level. Another 3 central stars are likely to be binaries (Douchin et al., in preparation).

The IR excess technique aims to detect the IR emission due to the presence of a cool companion. It relies on high precision optical and NIR photometry to detect the faintest possible companions. So far, the detection limit is an M8-type star for intrinsically faint central stars $\left(M_{V}=6-8\right)$. This technique is not biased by binary separation, so it is well suited to determine the binary fraction. We aim to perform high precision photometry for the entire $2 \mathrm{kpc}$ volume-limited sample of Frew (Frew 2008, see also Frew \& Parker 2007). So far, out of 28 central stars of PNe that were observed in the I-band, we have solidly detected an I-band excess in 3 with another possible 5 detections. The spectral types for the 3 detected companions are likely in the early $\mathrm{M}$ regime. We are as yet to quantify our survey's bias against fainter companions (Passy et al., in preparation).

\section{Current and future projects}

We are currently analysing additional $I$ and $J$-band photometry datasets which will provide us a preliminary estimate of the binary fraction (separations less than $500 \mathrm{AU}$ ).

In addition, space-based observations such as GAIA have a potential capability to detect the reflex orbital motion of central stars of $\mathrm{PNe}$ for binaries with intermediate separations and the extreme photometric precision of Kepler can be used to gauge how many close binaries have avoided detection in previous ground based surveys (see Douchin et al., these proccedings).

\section{References}

Bond, H. E. 2000, APNII, 199, 115

De Marco, O. 2009, PASP, 121, 316

Frew, D. J. 2008, PhD thesis, Macquarie University, 30, 490

Frew, D. J. \& Parker, Q. 2007, APN IV, 68

Jacoby, G. H. et al. 2010, PASA, 27, 156

Miszalski, B. et al. 2009, A $\mathscr{E} A, 505,249$ 